\title{
Incidence of Nausea and Vomiting Induced by Oxycodone Administered with Prochlorperazine in Japanese Cancer Patients
}

\author{
Takeshi Yamada, Yoshikazu Kanazawa, Yuto Aoki and Eiji Uchida
}

\begin{abstract}
Department of Gastrointestinal and Hepato-Billiary-Pancreatic Surgery, Nippon Medical School
Background: Nausea and vomiting are the most frequent side effects of opioids and may cause the opioids to be discontinued. New methods for preventing opioid-induced nausea can improve cancer pain management. Oxycodone is one of the most frequently used opioid used in Japan because patients receiving oxycodone report less nausea and vomiting than do patients receiving morphine. The reported incidence of oxycodone-induced nausea varies widely, although the true incidence remains unclear. As a first step toward preventing oxycodone-induced nausea, we aimed to determine the incidence of and risk factors for oxycodone-induced nausea and vomiting.
\end{abstract}

Methods: In this observational study, we analyzed a series of consecutive inpatients with cancer who received oxycodone with prochlorperazine as a preventive antiemetic agent. Oxycodone $(5 \mathrm{mg})$ was administered either at 08:00 and 20:00 or at 09:00 and 21:00, and prochlorperazine (5 mg) was also given at the same times for 5 days.

Results: Of the 145 enrolled patients, 138 were suitable for analysis. The incidence of nausea was $18.1 \%$, and that of vomiting was $5.8 \%$. The incidence of nausea was higher, but not to a significant degree, in women than in men $(P=0.07)$. Furthermore, the incidence of vomiting in women was equal to that in men $(P=0.28)$, whereas the incidences of both nausea $(P=0.99)$ and vomiting $(P=0.89)$ in elderly patients were equal to those in younger patients. In addition, the incidence of nausea $(P=0.52)$ and vomiting $(P=$ $0.91)$ in patients with digestive system cancer was equal to that of patients with non-digestive system cancer.

Conclusions: The incidence of nausea induced by oxycodone with prochlorperazine was $18.1 \%$ in opioid-naïve Japanese inpatients. Female sex may be a risk factor for oxycodone-induced nausea. These results suggest that a clinical study would require 314 participants (157 in each group) to decrease the incidence from $18 \%$ to $8 \%(10 \%$ decrease) with a new preventive treatment (alpha error $=0.05$, beta error $=0.2$ ). (J Nippon Med Sch 2015; 82: 100-105)

Key words: oxycodone, prochlorperazine, nausea, vomiting

\section{Introduction}

Nausea and vomiting, together with constipation and drowsiness, are the most frequent side effects of opioids ${ }^{1}$, and such side effects may even lead to the discontinuation of opioid use and compromise pain management ${ }^{2}$. Although many patients have opioid-induced nausea and vomiting, with approximately $10 \%$ reporting nausea as the most bothersome symptom ${ }^{3}$, antiemetics are often administered only when symptoms appears rather than prophylactically, because the incidence of opioid-induced nausea and vomiting is thought to be low ${ }^{1}$. On the other hand, preventing opioid-induced nausea can improve the treatment of cancer pain and enhance quality of life ${ }^{4}$. However, is the incidence of opioid-induced nausea and vomiting actually low? Is there no need to prevent opioid-induced nausea and vomiting?

To find answer these questions, we need to know the incidence of opioid-induced nausea and vomiting. Oxycodone is one of the most frequently used opioid in Japan because patients receiving oxycodone report less

Correspondence to Takeshi Yamada, Department of Gastrointestinal and Hepato-Billiary-Pancreatic Surgery, Nippon Medical School, 1-1-5 Sendagi, Bunkyo-ku, Tokyo 113-8603, Japan

E-mail: y-tak@nms.ac.jp

Journal Website (http://www.nms.ac.jp/jnms/) 
nausea and vomiting than do patients receiving morphine $^{5,6}$. According to previous studies, the incidence of oxycodone-induced nausea in patients with cancer who have not received any preventive antiemetic agent is $13.3 \%$ to $51.9 \% \%^{2,6-9}$. However, 4 of these 5 studies included fewer than 30 patients, and the results are controversial. The incidence derived from these 5 studies varies widely, and the true incidence remains unclear.

As a result we aimed to determine the incidence of oxycodone-induced nausea and vomiting. In Japan, prochlorperazine is usually administered to prevent opioidinduced nausea and vomiting ${ }^{10}$; thus, we intended to determine the incidence of nausea and vomiting induced by oxycodone when administered with prochlorperazine. In this observational study, the primary endpoint was to understand the incidence of nausea and vomiting, while the secondary endpoint was to identify risk factors for oxycodone-induced nausea and vomiting.

\section{Patients and Methods}

\section{Patients}

We prospectively evaluated a series of consecutive patients with cancer who were given controlled-release oxycodone with prochlorperazine from August 2007 through July 2011. Inclusion criteria were as follows: 1) a definitive diagnosis of cancer, 2) opioid naïvety, 3) inpatient status, 4) administration of oxycodone (up to $10 \mathrm{mg} /$ day) with prochlorperazine, 5) confirmed diagnosis of moderate-to-severe cancer pain (Numeric Rating Scale $\geq$ 4), 6) good oral intake, and 7) management with a clinical pathway. Exclusion criteria were as follows: 1) administration of antiemetic agents other than prochlorperazine, 2) schizophrenia or dementia, 3) paralytic ileus, 4) hepatic impairment, 5) severe renal dysfunction, 6) administration of other long-acting opioids during the study period, 7) undergoing chemotherapy, and 8) death within 3 days after starting oxycodone.

\section{Treatment}

We managed the patients with a clinical pathway to avoid missing medications and to facilitate observation. We administered oxycodone (5 mg) either at 08:00 and 20:00 or at 09:00 and 21:00 (total $10 \mathrm{mg}$ /day) and prochlorperazine $(5 \mathrm{mg})$ at the same times for 5 days. The recommended duration of treatment with prochlorperazine is approximately 1 week $^{8}$. The National Comprehensive Cancer Network (NCCN) guidelines indicate that prophylactic antiemetics, such as prochlorperazine, should be used only for patients with a history of opioidinduced nausea and vomiting. However, in Japan, many opioid-naïve patients are given prochlorperazine with oxycodone. Therefore, we decided that it was more appropriate to evaluate oxycodone-induced nausea in patients who were given concomitant prochlorperazine. Furthermore, we also administered magnesium sulfate (1 $\mathrm{g}$ /day) or a sennoside (12-24 mg/day) to prevent constipation. Although we administered nonsteroidal antiinflammatory drugs (NSAIDs) in many cases, we did not administer NSAIDs when the primary physician judged that doing so was harmful. The most frequently used NSAID was etodolac (400 mg/day), and the second most frequently used NSAID was loxoprofen sodium (180 mg/ day). Immediate-release oxycodone $(2.5 \mathrm{mg})$ was administered as an escape analgesic medication with the dose titrated according to the pain intensity.

If patients had sudden breakthrough pain more than twice a day during treatment, increasing the dose of the next administration was considered. For patients who were given $5 \mathrm{mg}$, the treatment dose could be directly increased to $10 \mathrm{mg}$. For patients who were given $\geq 10 \mathrm{mg}$, the treatment dose could be increased by $25 \%$ to $50 \%$ of the current dose at each increase. This dose titration was continued until adequate pain control was achieved.

Pain control was considered adequate when the following conditions were fulfilled: the dose every 12 hours was unchanged, immediate-release oxycodone was used fewer than 3 times per day, the dosing regimen for any nonopioids was unchanged, the patients rated their pain intensity with a Numeric Rating Scale score of $<4$, and any adverse events were tolerable.

\section{Sample Size}

On the basis of our previous retrospective study, we predicted that the incidence of nausea would be $15 \%$. Power calculations indicated that 137 patients were required as study participants when we set $95 \%$ confidence intervals (CIs) with a $6 \%$ error. We predicted that some participants would drop out. In total, 145 consecutive patients were enrolled.

\section{Evaluations}

We defined "nausea" as an unpleasant and emergent urge to vomit, "vomiting" as a reflexive expulsion of digestive contents, and "gastrointestinal distress" as abdominal discomfort and stomach pain. The patients were asked to assess their nausea, drowsiness, and gastrointestinal distress twice each day (morning and bedtime) and recorded "yes" or "no" with the assistance of research nurses for 5 days. To ensure that records were complete, every morning research nurses confirmed their records of the previous day. The patients were also asked twice 
Table 1 Patients background

\begin{tabular}{lc}
\hline Age & $67.2(33-94)$ \\
Male : Female & $93: 45$ \\
Elderly : Not elderly & $55: 83$ \\
Digestive tract : Non-digestive tract & $101: 37$ \\
\hline
\end{tabular}

Table 2 Primary site

\begin{tabular}{lrr}
\hline \multicolumn{1}{c}{ Primary site } & $\mathrm{N}$ & $\%$ \\
\hline Colorectal & 29 & 21.0 \\
Pancreas & 28 & 20.3 \\
Stomach & 26 & 18.8 \\
Liver and Bile duct & 14 & 10.1 \\
Urological & 12 & 8.7 \\
Hematological & 6 & 4.3 \\
Gynecological & 5 & 3.6 \\
Esophagus & 4 & 2.9 \\
Breast & 4 & 2.9 \\
Others & 10 & 7.2 \\
\hline
\end{tabular}

daily to assess the acceptability of therapy with consideration of their pain intensity during the previous 12hour period. Constipation was regarded as a stool-free interval of more than 72 hours during the 5 days. Vomiting was judged by the research nurses or investigators.

The primary endpoint of this study was the incidence and $95 \% \mathrm{CI}$ of nausea caused by administration of $10 \mathrm{mg}$ of oxycodone in opioid-naïve patients with cancer who were given prochlorperazine to prevent nausea and vomiting. The percentages of nausea and vomiting were analyzed with the Clopper-Pearson method and a 95\% CI. The secondary endpoint was the identification of risk factors for nausea and vomiting. The incidence rates of nausea and vomiting were compared by means of the chisquare test between men and women, between elderly ( $\geq$ 70 years of age) and younger patients, and between patients with digestive system cancer and those with nondigestive system cancer. A $P$ value of $<0.05$ was considered to indicate statistical significance. All analyses were performed with the software package IBM SPSS Statistics Base, version 20 (IBM Japan Ltd., Tokyo, Japan).

\section{Results}

A total of 163 patients were given oxycodone and managed with a clinical pathway. Eighteen patients were excluded because they were not receiving prochlorperazine or were given other preventive antiemetic agents on day 1. According to our plan, we enrolled 145 consecutive patients in this study. However, 2 patients were excluded
Table 3 Adverse events

\begin{tabular}{lrc}
\hline & $\mathrm{N}$ & $\%(95 \% \mathrm{CI})$ \\
\hline Constipation & 28 & $20.3(13.6-27.0)$ \\
Nausea & 25 & $18.1(11.6-24.5)$ \\
Vomiting & 8 & $5.8(1.9-9.7)$ \\
Sleepiness & 47 & $34.1(26.2-42.0)$ \\
Respiratory depression & 1 & $0.7(0-2.1)$ \\
Gastrointestinal distress & 3 & $2.2(0-5.4)$ \\
\hline
\end{tabular}

because prochlorperazine was not administered after day 2 , and 5 patients were excluded because they died within 3 days of starting oxycodone. A total of 138 patients were included, of which none had previously used another opioid.

The mean age of patients was 67.2 years (range, 33-94 years), and the male-to-female ratio was 93 : 45 (Table 1). The primary site of cancer was the digestive system in nearly three-quarters of the study patients (101 patients, $73.2 \%$; Table 2). The average administered dose on the last day of the study was $16.8 \mathrm{mg}$ (range, 10-60 mg), and about two-thirds $(66.8 \%)$ of patients treated with $10 \mathrm{mg} /$ day of oxycodone experienced a good analgesic effect for 5 days.

The most frequent adverse effect was sleepiness, with an incidence of $34.1 \%$ (95\% CI, 26.2-42.0; Table 2). The incidence of nausea was $18.1 \%$ (95\% CI, 11.6-24.5), and that of vomiting was $5.8 \%$ (95\% CI, 1.9-9.7; Table 3). The incidence of nausea was higher, but not to a significant degree, in women than in men, $(P=0.07$; Table 4). Moreover, the incidence of vomiting in women was equal to that of men $(P=0.28)$ (Table 4$)$, whereas the incidences of both nausea $(P=0.99)$ and vomiting $(P=0.89)$ in elderly patients were equal to those in younger patients (Table $4)$. The incidences of both nausea $(P=0.52)$ and vomiting $(P=0.91)$ in patients with digestive system cancer were equal to those in patients with non-digestive system cancer (Table 4).

\section{Discussion}

This study is, to our knowledge, the first to evaluate the incidence of oxycodone-induced nausea and vomiting when oxycodone is administered with prochlorperazine to opioid-naïve patients. The incidence of nausea was $18.1 \%$ (95\% CI, $13.9 \%-23.4 \%$ ), and that of vomiting was $5.8 \%$ (95\% CI, 3.8\%-7.8\%).

Several small-scale studies (with 20 to 27 patients) and one large-scale study (with 1,823 patients) have previously investigated oxycodone-induced nausea and vomiting $^{2,6-9}$. In these studies, the incidence of nausea was 
Table 4 Subgroup analysis

\begin{tabular}{lcccccc}
\hline & Nausea (+) & Nausea (-) & \multicolumn{3}{c}{ Vomiting (+) } & Vomiting (-) \\
\hline Male & $14.0(13)$ & $86.0(80)$ & \multirow{2}{*}{$=0.07$} & $4.3(4)$ & $95.7(89)$ & $\mathrm{P}=0.28$ \\
Female & $26.7(12)$ & $73.3(33)$ & & $8.9(4)$ & $91.1(41)$ & \\
\hline Elderly & $18.2(10)$ & $81.8(45)$ & \multirow{2}{*}{$\mathrm{P}=0.99$} & $5.5(3)$ & $94.5(52)$ & $\mathrm{P}=0.89$ \\
Not Elderly & $18.1(15)$ & $81.9(68)$ & & $6.0(5)$ & $94.0(78)$ & \\
\hline Digestive & $16.8(17)$ & $83.2(84)$ & \multirow{2}{*}{$\mathrm{P}=0.52$} & $5.9(6)$ & $94.1(95)$ & \multirow{2}{*}{$\mathrm{P}=0.91$} \\
Non-digestive & $21.6(8)$ & $78.4(29)$ & & $5.4(2)$ & $94.6(35)$ & \\
\hline
\end{tabular}

$13.3 \%$ to $51.9 \%$. Oxycodone-induced nausea and vomiting most often begins on the first administration of oxycodone to 5 days afterwards. To evaluate the exact incidence of oxycodone-induced nausea, we interviewed patients twice a day for 5 days because patients are sometimes not able to recall their experiences clearly. In contrast, patients in the largest previous study were evaluated every week for 8 weeks ${ }^{9}$. In any case, more than $10 \%$ of the patients with cancer who used oxycodone for pain relief had oxycodone-induced nausea. These findings suggest that a new preventive treatment for oxycodone-induced nausea is needed.

Female sex may be a risk factor for oxycodone-induced nausea. Indeed, the incidence of nausea in female patients was higher than that in male patients, although the difference was not significant $(\mathrm{P}=0.07)$. However, power calculations indicated that 348 patients (174 in each group) would be required to achieve a power of $90 \%$ with a 2-sided alpha value of 0.05 to demonstrate a sex difference. Therefore, this study was underpowered to validate the difference in this prevalence. A study including more than 1,000 participants found that opioids had a stronger analgesic effect in female patients than in male patients $^{11}$, suggesting that the adverse effects of opioids may also be stronger in female patients than in male patients.

The present study did not identify digestive system cancer as a risk factor for oxycodone-induced nausea and vomiting. We had expected digestive symptoms, including nausea and vomiting, to be amplified in patients with digestive system cancer. However, we found that the incidence of nausea and vomiting in patients with digestive system cancer was equal to that of patients with non-digestive system cancer. Furthermore, we had expected the rate of nausea and vomiting to be higher in elderly patients than in younger patients because the blood concentration of oxycodone may be higher in elderly patients than in younger patients. However, we found that the incidence of nausea and vomiting was equal in the 2 age groups. Thus, old age was also not a risk factor of oxycodone-induced nausea and vomiting.

An important factor in the development of oxycodoneinduced nausea and vomiting may be the timing of prochlorperazine administration. We administered the first dose of prochlorperazine at the same time as the first dose of oxycodone. Two hours may be required to achieve an effective blood concentration after the first administration of prochlorperazine. Williams and Smith have reported that prochlorperazine administered 1 hour earlier than morphine can reduce morphine-induced nausea $^{12}$. Therefore, the first dose of prochlorperazine should be administered 1 or 2 hours before the first dose of oxycodone.

Another important factor in the development of oxycodone-induced nausea and vomiting may be the dose of prochlorperazine. Although we administered 10 mg of prochlorperazine per day, many Japanese physicians administer $15 \mathrm{mg}$. To our knowledge, no study has evaluated the dose of prochlorperazine necessary to prevent nausea due to a controlled-release opioid. Williams and Smith have also reported that a divided dose of prochlorperazine (6 mg twice daily) was able to reduce nausea induced by morphine administered intravenously after surgery to English patients ${ }^{13}$. Because Japanese patients weigh less, on average, than English patients, 10 mg may be an adequate dose for them. Further study is required to determine whether a prochlorperazine dose of $15 \mathrm{mg}$ or more can induce a stronger antiemetic effect in patients with oxycodone-induced nausea.

Although several studies have investigated the efficacy of preventive antiemetic agents for opioid-induced nausea, none have been prospective or have involved the administration of individual preventive antiemetic agents to clarify their respective antiemetic effect. In a case control study, Ishihara et al. ${ }^{14}$ found that preventive antiemetic agents, including prochlorperazine, domperidone, and metoclopramide, can decrease the incidence of vomiting and nausea induced by various opioids (oxycodone, mor- 
phine, and codeine). However, in another retrospective study, Ishihara et al. found that preventive antiemetic agents could not reduce the incidence of opioid-induced nausea and vomiting ${ }^{15}$. They also used several different types of opioids and antiemetics but administered the preventive antiemetic agents after administering the opioid analgesics.

Our study is a first step toward successful prevention of oxycodone-induced nausea. Prospective interventional trials are required to identify the best preventive antiemetic agent. However, because the incidence of oxycodone-induced nausea is unclear; it is difficult to design an interventional study that evaluates the efficacy of preventive antiemetic agents. The results of our observational study suggest that a clinical study would require 314 participants (157 in each group) to decrease the incidence from $18 \%$ to $8 \%$ ( $10 \%$ decrease) with a new preventive treatment (alpha error $=0.05$, beta error $=0.2$ ).

Opioid-induced nausea is mediated by dopamine, histamine, and serotonin; therefore, their respective antagonists can prevent opioid-induced nausea ${ }^{1}$. Prochlorperazine cannot prevent nausea caused by histamine because prochlorperazine inhibits mainly dopamine receptors. In this study, nausea in approximately half of patients was alleviated by chlorpheniramine maleate, which inhibits histamine receptors (data not shown).

To reduce the incidence of opioid-induced nausea, agents that inhibit dopamine and histamine in a balanced manner are required. For example, olanzapine, which blocks multiple neurotransmitters, including dopaminergic, serotonergic, adrenergic, histaminergic, and muscarinic receptors ${ }^{16}$, is a strong candidate for future usage. In a ferret model, olanzapine decreased opioid-induced nausea and vomiting in a dose-dependent manner ${ }^{17}$. Coadministration of prochlorperazine, which inhibits dopamine receptors, and diphenhydramine, which inhibits histamine receptors, is another candidate for a balanced therapeutic effect. The results of the present study can serve as a baseline for new studies to develop therapies to prevent oxycodone-induced nausea.

This study has several limitations. First, our observations were conducted over a period of only 5 days. However, adverse events, including constipation, nausea, and vomiting, are noted most frequently in the first week and decrease over time ${ }^{18}$. Thus, it is unlikely that the short observation period caused the incidence of nausea to be underdiagnosed. Second, this study included only inpatients, who were managed by many physicians and nurses with adequate attention, rather than outpatients, who may have a higher incidence of nausea. Third, our study included only opioid-naïve patients who received only $10 \mathrm{mg} /$ day of oxycodone. Indeed, the incidence of nausea may be higher in patients given a higher dose of oxycodone.

In conclusion, the incidence of nausea induced by oxycodone administered with prochlorperazine was $18.1 \%$ in opioid-naïve Japanese inpatients. Female patients may be at a higher risk for oxycodone-induced nausea and vomiting. Studies with larger numbers of patients (300 or more) should be performed to evaluate new therapies for preventing oxycodone-induced nausea and vomiting. Equal numbers of male and female patients should be included in each treatment group, but less crucial are the ratio of elderly to younger patients and the ratio of patents with digestive system cancer to those with nondigestive system cancer.

\section{References}

1. McNicol E, Horowicz-Mehler N, Fisk RA, Kyle Bennett $\mathrm{K}$, Gialeli-Goudas M, Priscilla W, Chew PW, Lau J, Carr D: Management of opioid side effects in cancer-related and chronic noncancer pain: a systematic review. J Pain 2003; 4: 231-256.

2. Campora E, Merlini L, Pace M, Bruzzone M, Luzzani M, Gottlieb A, Rosso R: The incidence of narcotic-induced emesis. J Pain Symptom Manage 1991; 6: 428-430.

3. Cook SF, Lanza L, Zhou X, Sweeney CT, Goss D, Hollis $K$, Mangel AW, Fehnel SE: Gastrointestinal side effects in chronic opioid users: results from a population-based survey. Aliment Pharmacol Ther 2008; 27: 1224-1232.

4. Aparasu R, McCoy RA, Weber C, Mair D, Parasuraman TV: Opioid-induced emesis among hospitalized nonsurgical patients: effect on pain and quality of life. J Pain Symptom Manage 1999; 18: 280-288.

5. Lauretti GR, Oliveira GM, Pereira NL: Comparison of sustained-release morphine with sustained-release oxycodone in advanced cancer patients. Br J Cancer 2003; 89: 2027-2030.

6. Kalso E, Vainio A: Morphine and oxycodone hydrochloride in the management of cancer pain. Clin Pharmacol Ther 1990; 47: 639-646.

7. Heiskanen T, Kalso E: Controlled-release oxycodone and morphine in cancer related pain. Pain 1997; 73: 37-45.

8. Koizumi W, Toma H, Watanabe K, Katayama K, Kawahara M, Matsui K, Takiuchi H, Yoshino K, Araki N, Kodama K, Kimura H, Kono I, Hasegawa H, Hatanaka K, Hiraga K, Takeda F: Efficacy and tolerability of cancer pain management with controlled-release oxycodone tablets in opioid-naive cancer pain patients, starting with 5 mg tablets. Jpn J Clin Oncol 2004; 34: 608-614.

9. Yu SY: Postmarketing surveillance study of OxyContin tablets for relieving moderate to severe cancer pain. Oncology 2008; 74 (Suppl 1): 46-51.

10. Yomiya K, Matsuo N, Okuyama S: [Usefulness of perospirone for the prevention of nausea related to opioid administration]. Gan To Kagaku Ryoho 2008; 35: 625-628.

11. Pleym H, Spigset O, Kharasch ED, Dale O: Gender differences in drug effects: implications for anesthesiologists. 
Acta Anaesthesiol Scand 2003; 47: 241-259.

12. Williams PI, Smith M: An assessment of prochlorperazine buccal for the prevention of nausea and vomiting during intravenous patient-controlled analgesia with morphine following abdominal hysterectomy. Eur J Anaesthesiol 1999; 16: 638-645.

13. Morgan RJ Jr, Synold T, Carr BI, Doroshow JH, Womack EP, Shibata S, Somlo G, Raschko J, Leong L, McNamara M, Chow W, Tetef M, Margolin K, Akman S, Longmate J: Continuous infusion prochlorperazine: pharmacokinetics, antiemetic efficacy, and feasibility of high-dose therapy. Cancer Chemother Pharmacol 2001; 47: 327-332.

14. Ishihara M, Iihara H, Okayasu S, Yasuda K, Matsuura K, Suzui M, Itoh Y: Pharmaceutical interventions facilitate premedication and prevent opioid-induced constipation and emesis in cancer patients. Support Care Cancer 2010; 18: 1531-1538.

15. Ishihara $M$, Ikesue $H$, Matsunaga $H$, Suemaru $K$, Kitaichi K, Suetsugu K, Oishi R, Sendo T, Araki H, Itoh Y: A multi-institutional study analyzing effect of prophylactic medication for prevention of opioid-induced gastrointesti- nal dysfunction. Clin J Pain 2012; 28: 373-381.

16. Passik SD, Lundberg J, Kirsh KL, Theobald D, Donaghy K, Holtsclaw E, Cooper M, Dugan W: A pilot exploration of the antiemetic activity of olanzapine for the relief of nausea in patients with advanced cancer and pain. J Pain Symptom Manage 2002; 23: 526-532.

17. Torigoe K, Nakahara K, Rahmadi M, Yoshizawa K, Horiuchi $\mathrm{H}$, Hirayama S, Imai $\mathrm{S}$, Kuzumaki $\mathrm{N}$, Itoh $\mathrm{T}$, Yamashita A, Shakunaga K, Yamasaki M, Nagase H, Matoba M, Suzuki T, Narita M: Usefulness of olanzapine as an adjunct to opioid treatment and for the treatment of neuropathic pain. Anesthesiology 2012; 116: 159-169.

18. Pan H, Zhang Z, Zhang Y, Xu N, Lu L, Dou C, Guo Y, Wu S, Yue J, Wu D, Dai Y: Efficacy and tolerability of oxycodone hydrochloride controlled-release tablets in moderate to severe cancer pain. Clin Drug Investig 2007; 27: 259-267.

(Received, October 10, 2014)

(Accepted, January 8, 2015) 